\title{
Clinical Study \\ Study of the Influence of Upper Extremities Variation on Transradial Success
}

\author{
Saeed Yazdankhah, Ahmadreza Assareh, Mehdi Easapour, \\ Mohammad Nourizadeh, and Farzad Daeenejad \\ Cardiovascular Disease Research Center, Jondishapour University, Ahvaz 0098, Iran \\ Correspondence should be addressed to Ahmadreza Assareh; dr.assareh@gmail.com
}

Received 11 April 2013; Accepted 17 July 2013

Academic Editor: Kojiro Awano

Copyright ( 2013 Saeed Yazdankhah et al. This is an open access article distributed under the Creative Commons Attribution License, which permits unrestricted use, distribution, and reproduction in any medium, provided the original work is properly cited.

\begin{abstract}
Background. To investigate whether the presence of arterial anomalies in upper limb arteries can influence the success rate of transradial coronary angiography. Methods. retrograde transarterial sheath injection was done in patients with transradial coronary angiography. Arterial anomalies in the upper limb are evaluated in these patients. There are ten brachial (5.4\%), 7 radial (4/2\%), 5 subclavian (3\%), and 3 brachiocephalic arteries (1.8\%). There also were 4 loops in ulnar artery (2.4\%) and one in radial (0.6\%). Except cannulation time $(P=0.97)$, there were associations between anomalies and other times $(P=0.001)$ and contrast volume used $(P=0.009)$. Anomalies did not have any effect on procedural success rate and just in one patient because of subclavian loop,procedure changed to femoral approach $(P=0.19)$. Discussion. Patients without upper extremities anomalies in comparison with patients with anomalies had significantly shorter periods for angiographic time, catheterization time, and fluoroscopic time. We recommend femoral catheterization instead of radial catheterization in patients with upper extremities anomalies.
\end{abstract}

\section{Introduction}

Coronary angiography and interventions from the radial artery have gained popularity mainly because of a very low complication rate with immediate postprocedural sheath removal despite full heparinization and antiplatelet therapy [1]. The transradial approach (TRA) was also preferred by patients when compared to the transfemoral approach [2]. However, this approach has also some limitations, which include definite learning curve, procedural failure rate, and occasional patient discomfort caused by arterial spasm. These last two limitations are seen more frequently with radioulnar loops and extreme arterial tortuosity. Recently, dramatic reduction of pain and spasm has been described with the use of a hydrophilic-coated introducer sheath [3]. The transradial technique is, however, associated with a significant learning curve even for experienced femoral operators [4-6]. Although procedure failures can sometimes be due to radial artery anatomical variations, there are limited data describing such variations [7]. We undertook the establishment of the frequency of radial artery anomalies and their relation to procedure outcome in patients undergoing a first transradial coronary procedure.

\section{Materials and Methods}

2.1. Study Population. This was a prospective study that was performed in Imam Hospital of Iran. A total of 164 consecutive patients undergoing their first transradial coronary procedure were recruited from May 2011 to December 2011. Only patients undergoing diagnostic angiography and coronary intervention were studied. Clinical characteristics, procedural information, and clinical outcomes were obtained from the database and confirmed by chart review. Patients with a previous transradial procedure were excluded. 


\subsection{Radial Artery Cannulation}

\subsubsection{Transradial Procedure}

Right TRA. After positive evaluation of radioulnar hand arterial arch patency with oximetry and plethysmography test [4], the right arm was placed without constraint in an abducted position with slight wrist overextension [5]. Local skin anesthesia was obtained with $0.2 \mathrm{cc}$ of lidocaine $2 \%$ injected subdermally with a 20 gauge needle. A small $(1 \mathrm{~mm})$ incision was made with no. 11 surgical blade before radial artery was punctured at approximately $2 \mathrm{~cm}$ proximal to the styloid process with either a 20 gauge open needle to obtain a pulsatile blood flow. The artery was cannulated with either a guidewire. A 4-6 Fr, hydrophilic sheath was then inserted. The arm was then positioned alongside the patient and an arterial vasodilator (containing $100 \mu \mathrm{g}$ nitroglycerine and $2.5-5.0 \mathrm{mg}$ of verapamil) was given according to local protocols. Heparin (2,500-5,000 iu.) was given either as part of the vasodilator cocktail or in the aortic root.

2.2.2. Retrograde Radial Arteriography. Retrograde radial arteriography was performed following administration of the arterial vasodilator to define radial artery anatomy from mid radius to radio-brachial anastomosis. A solution of $4 \mathrm{mls}$ of contrast mixed with $8 \mathrm{mls}$ of blood (to dilute the contrast and minimise any discomfort from contrast injection) was injected briskly through the side arm of the sheath with radiographic acquisition at the elbow in an anteroposterior projection. If a high-bifurcating radial origin was identified, a further arteriogram was obtained higher up the arm to identify the point of anastomosis to the brachial artery.

2.2.3. Transradial Coronary Procedures. Retrograde radial arteriography was performed prior to coronary intubation in all patients. In patients who had a failed transradial puncture due to hematoma, it was at the discretion of the operator to attempt the contralateral radial artery or use the transfemoral approach. The arterial sheath was removed immediately after completion of the transradial procedure and haemostasis was achieved using a unilateral radial compression system.

2.3. Classifications and Definitions. The site of anomalous origin was determined with reference to the intercondylar line of the humerus, which is a fixed line representing the proximal border of the antecubital fossa.

Bifurcation of the brachial artery proximal to this line is considered a variant pattern.

A radial artery loop was defined as the presence of a full $360^{\circ}$ loop of the radial artery distal to the bifurcation of the brachial artery. Extreme radial tortuosity was defined as the presence of a bend of more than $90^{\circ}$ in the contour of the vessel. Anatomical variations that did not fit into these specified categories were grouped together and categorised as "other" anomalies.

Angiographic duration was defined as time interval when coronary cannulation was performed until the last image was taken. Procedural success was defined as completion of the planned procedure via the initially selected radial access route. "Access time" was defined as the time interval from administration of local anaesthesia at the arterial puncture site to successful placement of an arterial sheath. "Cannulation time" was defined as the time interval from placement of the arterial sheath to successful acquisition of the first angiographic image. Minor vascular complications were defined as haematoma $<5 \mathrm{~cm}$, vessel dissection without ensuing ischaemia, pseudoaneurysm, and localised infection. Major vascular complications were defined as haematoma $>5 \mathrm{~cm}$, any access site complications that required surgical or radiological intervention, $>3 \mathrm{gm} / \mathrm{dL}$ haemoglobin drop due to access site bleeding, bleeding requiring transfusion, limb ischaemia, and/or compartment syndrome.

2.4. Data Collection. Patient demographics, procedural data, and radial arteriography findings with specific details of any anomalies and local vascular complications were collected on a specifically written data management database.

2.5. Statistical Analysis. Statistical analysis was performed using SPSS 16.0 for Windows (SPSS Inc., Chicago, Illinois, USA). Categorical data were presented as absolute values and percentages whereas continuous data were presented as mean \pm standard deviation. Student's $t$ test was used to compare continuous data as appropriate.

2.6. Categorical Data. Categorical data were compared using the chi-square test with the appropriate degree of freedom. Multivariate regression analysis was used to examine potential correlation between radial artery anomaly and variables such as sex, age, hypertension, and diabetes, and a $P$ value of less than 0.05 was considered to be statistically significant.

\section{Results}

3.1. Patients and Procedural Characteristics. A total of 164 patients were studied with mean age $567 \pm 11 / 1$ years and $70.3 \%$ male.

Baseline patient and procedural data are summarised on Tables 1 and 2.

Transradial procedural success was $96.6 \%$ with $3.4 \%$ of patients requiring femoral access for procedure completion.

3.2. Radial Artery Anatomy and Procedural Outcome. There were 2 cases $(1.4 \%)$ of radial puncture failure. The failure of puncture was mainly due to hematoma in the site of puncture. Retrograde radial arteriography was obtained in 164 patients. Anomalies were noted in 44 (26.7\%) and there was not significant change in age (mean age 59.3 years versus 57.7, $P>0.05$ ) more commonly female (36\% versus $28 \%, P=$ 0.02 ) with significantly higher procedure failure (Table 3 ). Although procedure duration and fluoroscopy time were longer in patients with anomalies, these were not statistically significant.

3.3. Normal Radial Artery Anatomy. Normal radial artery anatomy was present in 138 patients (86.2\%). 
TABLE 1: Basic characteristics of patients.

\begin{tabular}{lcc}
\hline & Number & Percent (\%) \\
\hline Age & $56.7 \pm 11 / 1$ & \\
Male & 116 & $70 / 3$ \\
Smoker & 43 & $26 / 1$ \\
HTN & 74 & $44 / 8$ \\
HLP & 52 & $31 / 5$ \\
DM & 34 & $20 / 6$ \\
Normal coronary & 41 & $27 / 7$ \\
(1) Vessel disease & 24 & $16 / 7$ \\
(2) Vessel disease & 34 & $22 / 9$ \\
(3) Vessel disease & 46 & 31 \\
Left main & 8 & $5 / 4$ \\
More than 1 catheter & 36 & 25 \\
Anomaly & 44 & $26 / 7$ \\
Spasm of radial artery & 33 & $22 / 3$ \\
\hline
\end{tabular}

TABLE 2: Ages of male and female patients.

\begin{tabular}{lccccc}
\hline Number & $\begin{array}{c}\text { Minimum } \\
\text { (years) }\end{array}$ & $\begin{array}{c}\text { Maximum } \\
\text { (years) }\end{array}$ & $\begin{array}{c}\text { Mean } \\
\text { (years) }\end{array}$ & $\begin{array}{c}\text { Standard } \\
\text { deviation }\end{array}$ \\
\hline Male & 116 & 30 & 86 & $57 / 2$ & $11 / 7$ \\
Female & 49 & 36 & 75 & $55 / 4$ & $9 / 4$ \\
Total & 165 & 30 & 86 & $56 / 7$ & $11 / 1$ \\
\hline
\end{tabular}

TABLE 3: Number of male and female patients with and without radial anomaly.

\begin{tabular}{lccc}
\hline & Male & Female & Total \\
\hline \multirow{2}{*}{ Without anomaly } & 88 & 33 & 121 \\
& $72 / 7 \%$ & $27 / 3 \%$ & $100 \%$ \\
With anomaly & 28 & 16 & 44 \\
& $63 / 6 \%$ & $36 / 4 \%$ & $100 \%$ \\
\hline \multirow{2}{*}{ Total } & 116 & 49 & 165 \\
& $70 / 3 \%$ & $29 / 7 \%$ & $100 \%$ \\
\hline
\end{tabular}

Procedure failures were due to subclavian artery loop (1 patient).

3.4. Radial Anomalies. A summary of anomaly types and associated failure rates is shown in Figure 1 and Table 3 . There were no relations seen between age and gender and presence of radial artery anomaly $(P>0.05)$.

3.5. High-Bifurcating Radial Origin. This was the most frequent radial anomaly (Figures $2(\mathrm{a})$ and $2(\mathrm{~b})$ ) observed in 8 patients with a frequency of $4.8 \%$. The majority of these vessels rejoined the brachial artery at the level of mid or upper humerus.

High-bifurcating radial artery was not associated with a high incidence of transradial failure $(P>0.05)$.

3.6. Extreme Radial Artery Tortuosity. Extreme radial artery tortuosity (Figure 3) was observed in 7 patients (4.2\%).

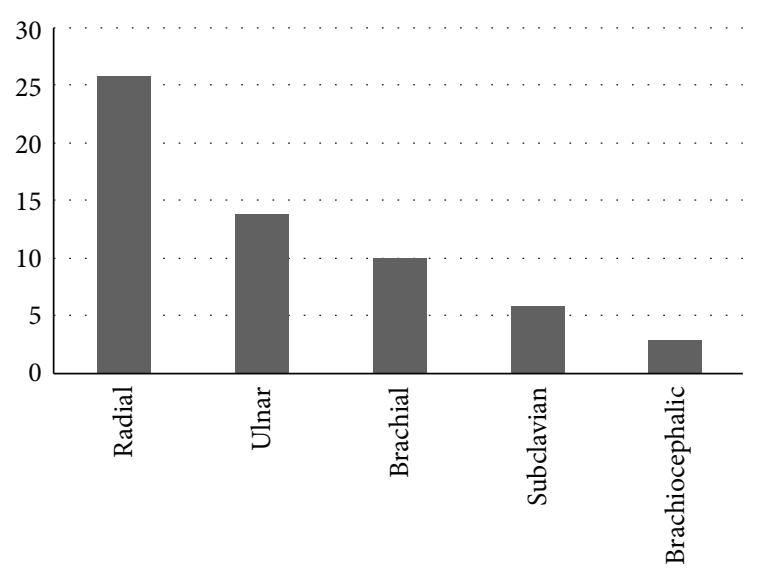

FIGURE 1: Number of anomalies in each artery.

Although these vessels were prone to severe radial artery spasm, the presence of extreme radial tortuosity was not associated with a high procedural failure rate.

These had no clinical significance since all cases were completed via the chosen radial access site.

3.7. Radial Artery Loop. A radial artery loop (Figure 4) was observed in 1 patient $(0.6 \%)$. This type of anomaly mostly involved the proximal radial artery just below the brachial bifurcation.

3.8. Vascular Complications. No patients had bleeding requiring transfusion or surgical intervention. Access site vascular complications in 3 patients (2\%) were treated conservatively. These were large haematoma (2), arteriovenous fistula (1), immediate radial artery occlusion (2), and axillary artery dissection (1). Two patients with large haematoma were successfully treated with customised compression and arm elevation without evidence of compartment syndrome or hand ischaemia. The patient with arteriovenous fistula was also managed conservatively without ischaemic sequelae.

3.9. Procedural Data. The mean duration of puncture time, catheter time, angiographic time, fluoroscopy time, and volume contrast in all patients (Table 4) and patients with and without radial artery anomaly are shown (Table 5 ).

There was no significant change in the time of puncture between patients with and without anomalies.

But catheter time, angiographic time, fluoroscopic time, and the volume contrast used were significantly further than those in patients without anomalies $(P<0.05)$.

\section{Discussion}

Transradial percutaneous coronary procedures have gained popularity because of reduced access site vascular complications and immediate patient mobilization [8]. Procedural success has been facilitated through technological enhancements. In this study the time of puncture between 


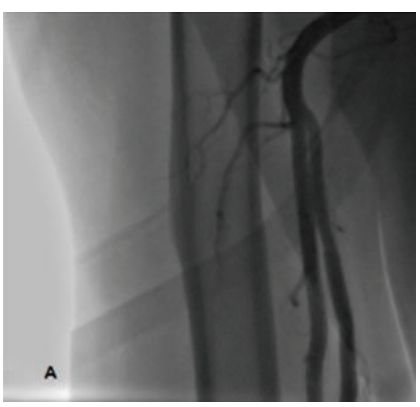

(a)

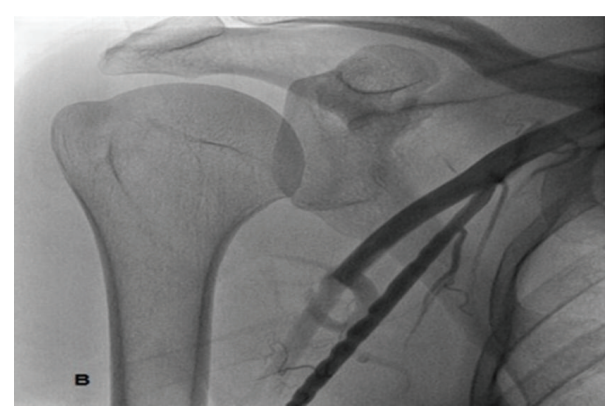

(b)

FiguRE 2: (a) High bifurcation of radial artery. (b) High bifurcation of radial artery.

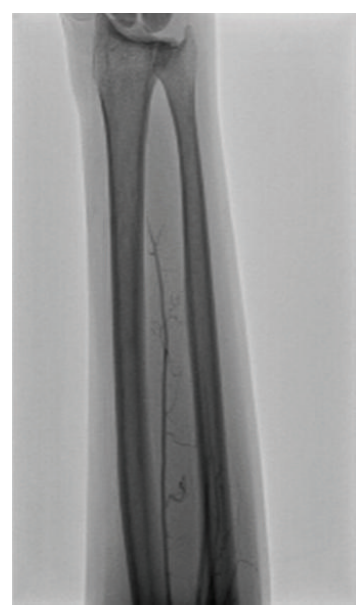

FIgURE 3: Radial hypoplasia.

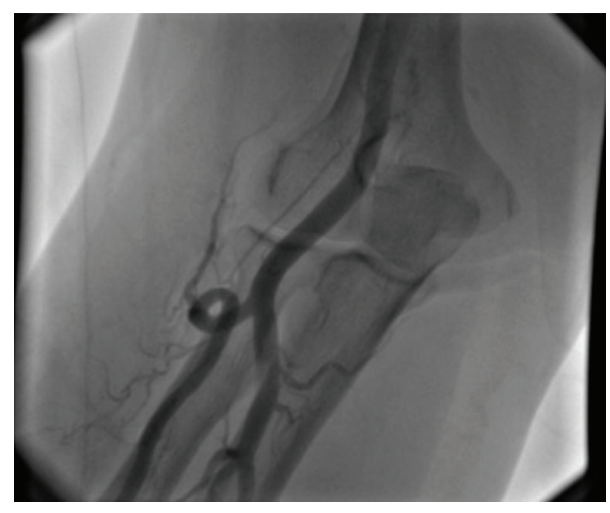

FIgURE 4: Radial loop.

patients with and without anomalies didn't change significantly because two patients with inability to puncture due to hematoma were excluded from study and other anomalies seems higher than puncture time and didn't effect in the puncture time.

But the time of passing catheter to aorta, angiographic time, fluoroscopic time, and volume contrast used were significantly more prolong than patients without anomalies
TABLE 4: Variation in time and volume contrast in all patients.

\begin{tabular}{lcccc}
\hline & $\begin{array}{c}\text { Minimum } \\
(\mathrm{s})\end{array}$ & $\begin{array}{c}\text { Maximum } \\
(\mathrm{s})\end{array}$ & $\begin{array}{c}\text { Mean } \\
(\mathrm{s})\end{array}$ & $\begin{array}{c}\text { Standard } \\
\text { deviation }\end{array}$ \\
\hline Puncture time & 17 & 300 & 60 & 50 \\
Catheter time & 7 & 300 & 35 & 40 \\
Angiography time & 100 & 2250 & 370 & 320 \\
Fluoroscopy time & 44 & 1500 & 210 & 190 \\
$\begin{array}{l}\text { Volume of contrast } \\
\text { agent }(\mathrm{cc})\end{array}$ & 25 & 120 & 40 & 14 \\
\hline
\end{tabular}

mainly due to prolongation of passing guiding catheter through aorta to find the correct passage.

Reported technical failure for transradial procedures is between 1 and 5\% [9-11] compatible with our overall figure of $3.4 \%$. There are several reasons leading to failure: inability to puncture, artery spasm, and anatomical variations.

Autopsy studies of upper-limb arterial anatomical variation reported a frequency of between 4 and 18.5\% [11-14] In our study, the presence of radial abnormality was $26.6 \%$.

Not only were there wide variations in the occurrence of anomaly, but also there were also variations in the pattern of anomalies reported, partly due to differences in definitions. The frequency of anatomical variation was higher in autopsy studies as variation was defined according to the course of the artery in relation to muscle and nerve that would not be evident in arteriographic studies.

An interesting observation from the autopsy studies was an absence of radial artery tortuosity or hypoplasia. It is also worth noting that radial artery loop was not a separate category but under the category of variation of anastomosis between brachial artery and radial or ulnar artery at elbow level.

The most frequent radial artery anomaly observed is high radial bifurcation with a reported frequency range of $0 \%$ to $14.3 \%$. The absence of high radial bifurcation was reported using ultrasonographic scanning suggesting that it is not reliable in identifying this type of anomaly [14].

All loops were accompanied by a recurrent radial artery at the apex of the loop which invariably assumed a straight path into the upper arm. The presence of the remnant recurrent radial artery has potential to complicate the crossing and 
TABLE 5: Variations in time and volume contrast in patients with and without radial artery anomalies.

\begin{tabular}{lcccccccc}
\hline & \multicolumn{2}{c}{ Minimum (s) } & \multicolumn{2}{c}{ Maximum (s) } & \multicolumn{2}{c}{ Mean (s) } & \multicolumn{2}{c}{ Standard deviation } \\
\hline Anomaly & + & - & + & - & + & - & + & - \\
Puncture time & 20 & 17 & 240 & 300 & 70 & 60 & 60 & 50 \\
Catheter time & 10 & 7 & 300 & 180 & 60 & 30 & 75 & 23 \\
Angiographic time & 130 & 100 & 2000 & 2250 & 590 & 315 & 510 & 230 \\
Fluoroscopic time & 60 & 45 & 1500 & 720 & 370 & 180 & 350 & 0.7 \\
Volume of contrast agent & 250 & 25 & 202 & 120 & 48 & 38 & 20 & 0.001 \\
\hline
\end{tabular}

straightening manoeuvre, with a tendency for the wire to selectively "follow" the path of the remnant artery, thereby increasing risk of dissection or perforation especially if such anatomy has not been initially defined.

Although loops can often be crossed using either a hydrophilic wire or an angioplasty wire and then straightened with a 5F JR4 configuration diagnostic catheter, these manoeuvres can induce spasm and pain, making subsequent catheter manipulation and advancement impossible.

However, more than half of these were patients with large diameter radial loop anatomy deemed impassable after radial arteriography, and procedures were completed via alternative access sites without attempting to cross the loops.

Our definition for extreme tortuosity differed from other studies with a consequent lower frequency of $2.0 \%$, compared to $3.8 \%, 4.2 \%$, and $5.2 \%$ by Yokoyama et al., Yoo et al., and Valsecchi et al., respectively [14-16].

Variations in bifurcation anatomy had no influence on procedural outcome.

The acquisition of a radial arteriogram requires only a minimal contrast load, a small amount of additional radiation, and trivial extra procedural time. This is offset by the provision of important information that aids the operator in planning an optimal procedure.

\section{Conclusion}

Radial artery anomalies are relatively common and a cause of transradial procedure failure even for experienced radial operators. Retrograde radial arteriography helps to delineate underlying anomalies and identify patients with unfavourable anatomy, thereby informing the operator to plan a strategy to overcome the anomaly or change access route with the potential to save time and avoid vascular complications. This can be performed with a minimum of contrast and should be considered part of a routine transradial procedure.

It is also clear that in patients without upper extremities anomalies in comparison with patients with anomalies, angiographic time, catheterization time, fluoroscopic time, and volume contrast used were significantly lower, and in this subgroup of patients we recommend femoral catheterization instead of radial catheterization.

\section{References}

[1] F. Kiemeneij, G. J. Laarman, D. Odekerken, T. Slagboom, and R. van der Wieken, "A randomized comparison of percutaneous transluminal coronary angioplasty by the radial, brachial and femoral approaches: the access study," Journal of the American College of Cardiology, vol. 29, no. 6, pp. 1269-1275, 1997.

[2] C. J. Cooper, R. A. El-Shiekh, D. J. Cohen et al., "Effect of transradial access on quality of life and cost of cardiac catheterization: a randomized comparison," American Heart Journal, vol. 138, no. 3, pp. 430-436, 1999.

[3] J. P. Dery, S. Simard, and G. R. Barbeau, "Reduction of discomfort at sheath removal during transradial coronary procedures with the use of a hydrophilic-coated sheath," Catheterization and Cardiovascular Interventions, vol. 54, no. 3, pp. 289-294, 2001.

[4] Y. Louvard, T. Lefevre, and M. C. Morice, "Radial approach: what about the learning curve?" Catheterization and Cardiovascular Diagnosis, vol. 42, no. 4, pp. 467-468, 1997.

[5] Y. Louvard, M. Pezzano, L. Scheers et al., "Coronary angiography by a radial artery approach: feasibility, learning curve. One operator's experience," Archives des Maladies du Coeur et des Vaisseaux, vol. 91, no. 2, pp. 209-215, 1998.

[6] S. L. Goldberg, R. Renslo, R. Sinow, and W. J. French, "Learning curve in the use of the radial artery as vascular access in the performance of percutaneous transluminal coronary angioplasty," Catheterization and Cardiovascular Diagnosis, vol. 44, no. 2, pp. 147-152, 1998.

[7] Y. Louvard and T. Lefevre, "Loops and transradial approach in coronary diagnosis and intervention," Catheterization and Cardiovascular Interventions, vol. 51, no. 2, pp. 250-252, 2000.

[8] J. P. Uglietta and S. Kadir, "Arteriographic study of variant arterial anatomy of the upper extremities," CardioVascular and Interventional Radiology, vol. 12, no. 3, pp. 145-148, 1989.

[9] M. Rodríguez-Niedenführ, T. Vázquez, L. Nearn, B. Ferreira, I. Parkin, and J. R. Sañudo, "Variations of the arterial pattern in the upper limb revisited: a morphological and statistical study, with a review of the literature," Journal of Anatomy, vol. 199, part 5, pp. 547-566, 2001.

[10] P. F. Ludman, N. G. Stephens, A. Harcombe et al., "Radial versus femoral approach for diagnostic coronary angiography in stable angina pectoris," American Journal of Cardiology, vol. 79, no. 9, pp. 1239-1241, 1997.

[11] G. R. Barbeau, "Radial loop and extreme vessel tortuosity in the transradial approach: advantage of hydrophilic-coated guidewires and catheters," Catheterization and Cardiovascular Interventions, vol. 59, no. 4, pp. 442-450, 2003.

[12] D. J. R. Hildick-Smith, P. F. Ludman, M. D. Lowe et al., "Comparison of radial versus brachial approaches for diagnostic coronary angiography when the femoral approach is contraindicated," American Journal of Cardiology, vol. 81, no. 6, pp. 770-772, 1998.

[13] A. Rodriguez-Baeza, J. Nebot, B. Ferreira et al., "An anatomical study and ontogenetic explanation of 23 cases with variations in 
the main pattern of the human brachio-antebrachial arteries," Journal of Anatomy, vol. 187, part 2, pp. 473-479, 1995.

[14] N. Yokoyama, S. Takeshita, M. Ochiai et al., "Anatomic variations of the radial artery in patients undergoing transradial coronary intervention," Catheterization and Cardiovascular Interventions, vol. 49, no. 4, pp. 357-362, 2000.

[15] B. S. Yoo, J. Yoon, J. Y. Ko et al., "Anatomical consideration of the radial artery for transradial coronary procedures: arterial diameter, branching anomaly and vessel tortuosity," International Journal of Cardiology, vol. 101, no. 3, pp. 421-427, 2005.

[16] O. Valsecchi, A. Vassileva, G. Musumeci et al., "Failure of transradial approach during coronary interventions: anatomic considerations," Catheterization and Cardiovascular Interventions, vol. 67, no. 6, pp. 870-878, 2006. 


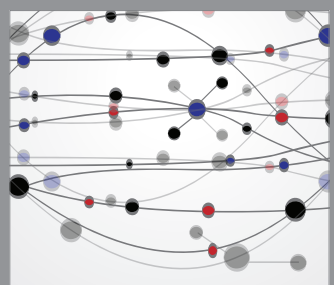

The Scientific World Journal
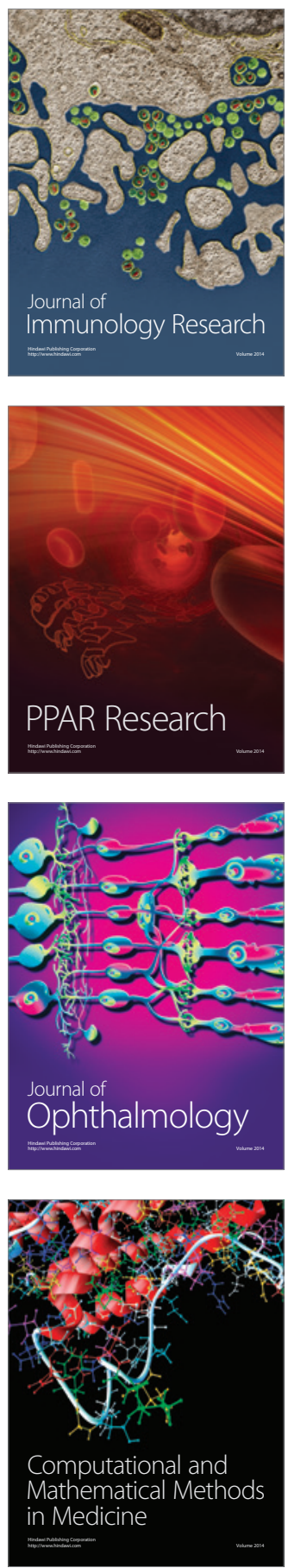

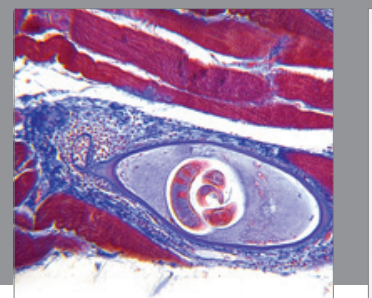

Gastroenterology

Research and Practice
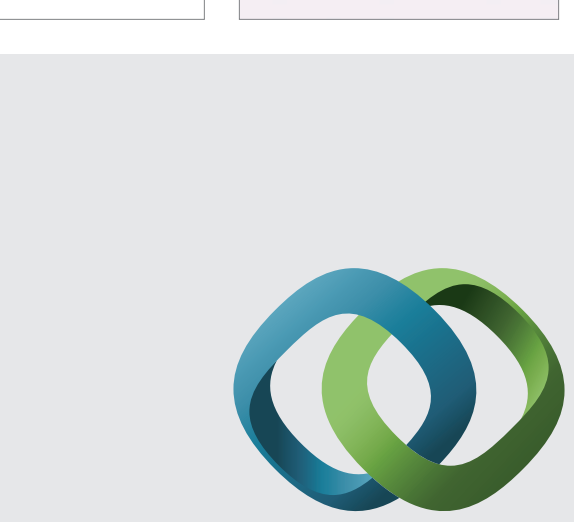

\section{Hindawi}

Submit your manuscripts at

http://www.hindawi.com
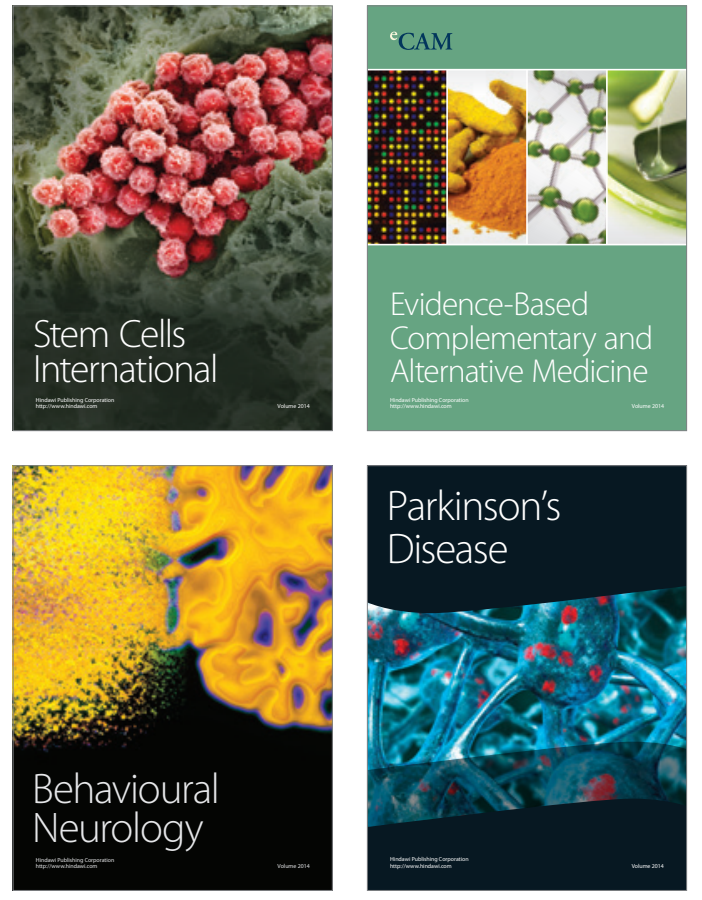
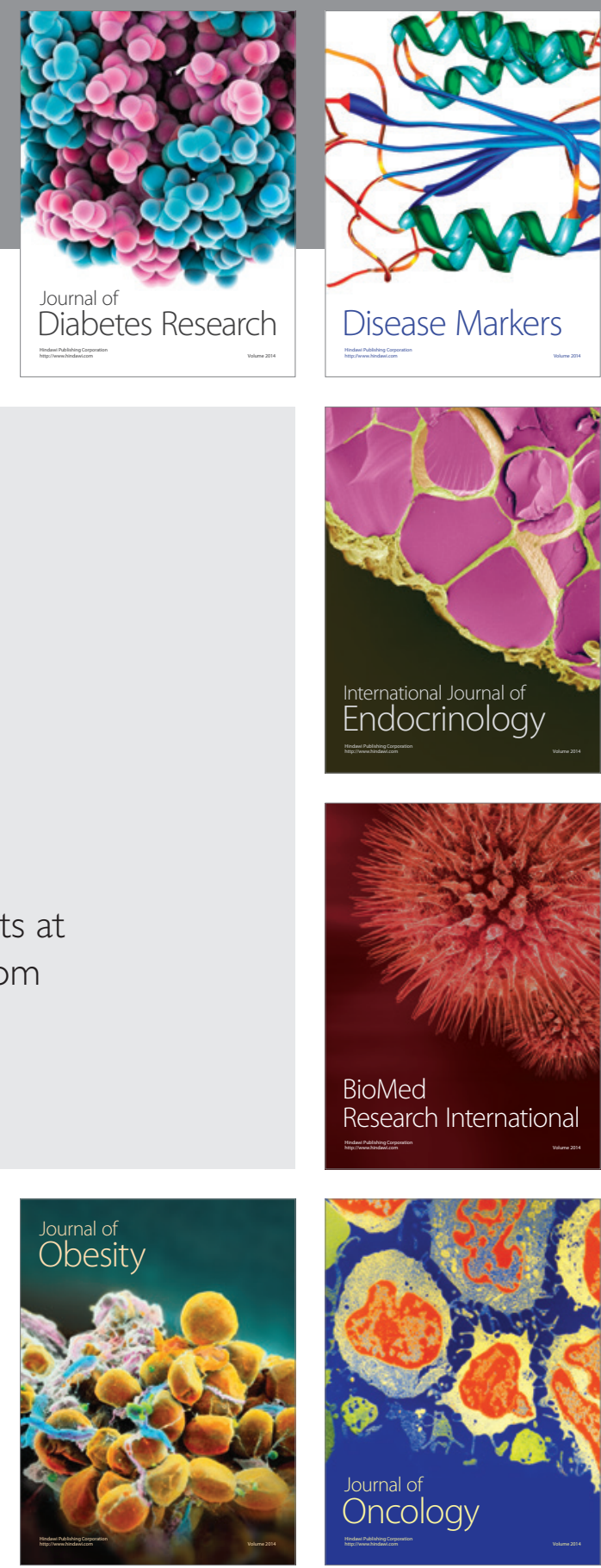

Disease Markers
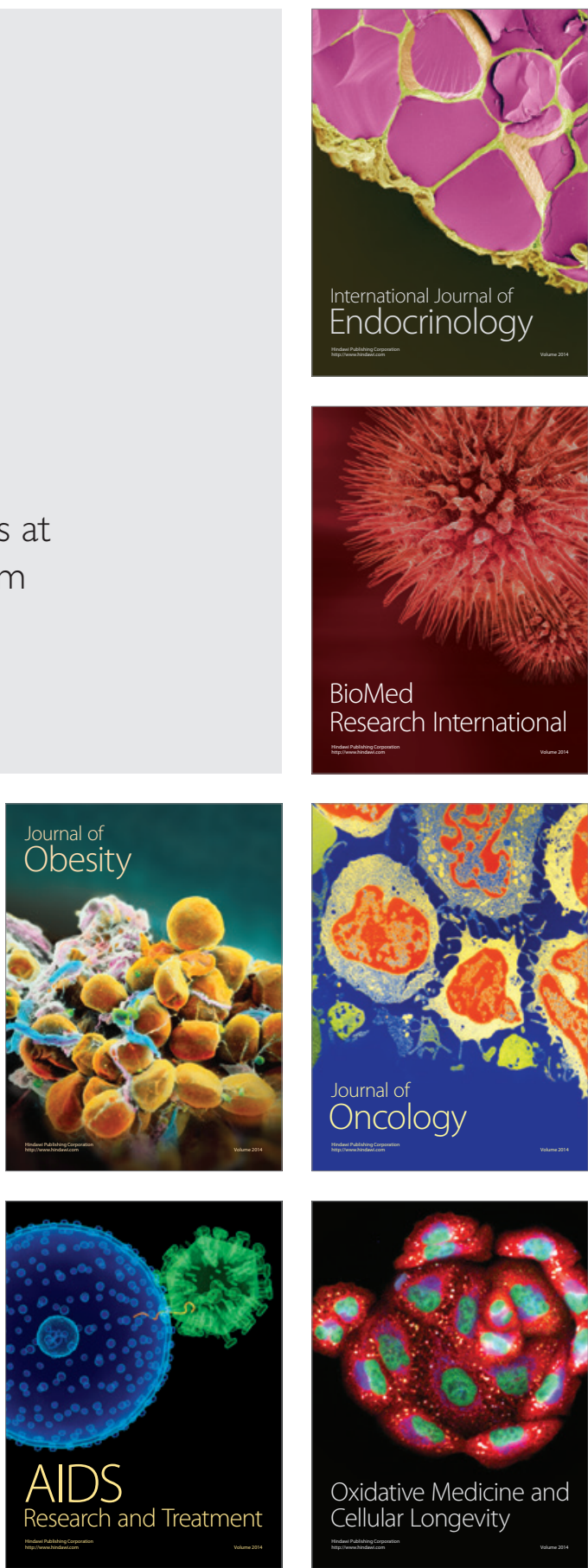